\title{
Electro-Optical Properties of InAs/GaSb Superlattice Infrared Photodiodes for Bispectral Detection
}

\author{
$\underline{A}$. Wörl $^{1,{ }^{*}}$, F. Rutz ${ }^{1}$, R. Rehm ${ }^{1}$, J.-M. Masur ${ }^{1}$, P. Kleinow ${ }^{1}$, J. Schmitz ${ }^{1}$, J. Niemasz ${ }^{1}$, W. Luppold ${ }^{1}$, \\ T. Stadelmann ${ }^{1}$, M. Walther ${ }^{1}$, T. Simon $^{2}$, R. Scheibner ${ }^{2}$ and J. Ziegler ${ }^{2}$ \\ ${ }^{1}$ Fraunhofer-Institut für Angewandte Festkörperphysik, Tullastraße 72, 79108 Freiburg, Germany \\ ${ }^{2}$ AlM Infrarot-Module GmbH, Theresienstraße 2, 74072 Heilbronn, Germany \\ *Corresp. author: andreas.woerl@iaf.fraunhofer.de
}

\begin{abstract}
:
InAs/GaSb short-period superlattices have proven their large potential for high-performance focal plane array infrared detectors. They are fabricated as monospectral and bispectral infrared detectors for a wavelength range between 3-30 $\mu \mathrm{m}$ with very high responsivity and thus comparable to state-ofthe-art $\mathrm{CdHgTe}$ and InSb detectors. Dual-color, mid-wavelength infrared InAs/GaSb superlattice camera systems offer simultaneous and spatially coincident detection on a millisecond time scale in both spectral channels between 3-4 $\mu \mathrm{m}$ and 4-5 $\mu \mathrm{m}$. Thus, these cameras are very sensitive to the spectral signature of carbon dioxide at approximately $4.3 \mu \mathrm{m}$ and can be used for remote imaging of $\mathrm{CO}_{2}$. In a molecular beam epitaxy based process, eleven $288 \times 384$ dual-color detector arrays are fabricated on 3" GaSb substrates. Very homogeneous detector arrays with an excellent noise equivalent temperature difference have been realized. This article presents the InAs/GaSb type-II superlattice dual-color concept, its results, and delivers insights into a range of test methodologies which ensure that the basic requirements for achieving high detector performance are fulfilled.
\end{abstract}

Key words: InAs/GaSb type-Il superlattice, bispectral infrared imager, carbon dioxide detection, focal plane array, electro-optical characterization.

\begin{abstract}
Introduction
Infrared (IR) photodetectors for the atmospheric transmission window in the mid-wavelength IR (3-5 $\mu \mathrm{m}, \mathrm{MWIR})$ and the long-wavelength IR (8-12 $\mu \mathrm{m}$, LWIR) are either based on small band gap semiconductors or quantum functional semiconductor heterostructures. The most prominent bulk materials with a bandgap in the MWIR are InSb and CdHgTe and for the LWIR spectral range CdHgTe. The fabrication of large $\mathrm{CdHgTe}$ arrays with homogeneous performance gets more and more challenging with longer cutoff wavelength. Alternative approaches, utilizing quantum confinement effects in tailored semiconductor heterostructures based on III-V compound semiconductors, have been proven to be very attractive for the development of highperformance IR detectors [1].
\end{abstract}

Quantum well infrared photodetector (QWIP) thermal imaging systems with high thermal resolution and excellent uniformity of focal plane arrays (FPAs) are meanwhile well established. However, in QWIPs normal incident absorption is forbidden by quantum mechanical selection rules, which require polarization of the incident radiation with a component perpendicular to the layers. Hence, the major drawback of QWIPs remains their low quantum efficiency (QE). For applications requiring a high frame rate or under very low photon flux conditions, QWIP detectors are not the technology of choice.

Short-period InAs/GaSb superlattices (SLs), as proposed by Smith and Mailhiot [2], represent a very attractive quantum system for highperformance infrared detectors $[3,4]$. They reveal a high effective electron mass, resulting in low tunneling currents, and promise lower Auger recombination rates. InAs/GaSb SLs are characterized by their broken gap type-II band alignment. The effective band gap $E_{g}$ can be tailored from $0.3 \mathrm{eV}$ to values below $0.1 \mathrm{eV}$ by varying the thickness of the InAs and $\mathrm{GaSb}$ layers. InAs/GaSb SLs for mid-IR detection usually consist of InAs and GaSb layers with a thickness between five and fifteen monolayers. The holes are confined in GaSb layers, whereas electron wave functions overlap considerably from one InAs layer to the adjacent InAs layers. The overlap of electron wave functions results in the formation of an electron miniband in the conduction band. The 
optical transitions between localized holes in GaSb layers and the electron miniband lead to the detection of infrared photons. Photodiodes (PDs) fabricated with InAs/GaSb SLs also offer high QE, making the material system very well suited for applications with high frame rates and short integration times. Soon after the demonstration of the first fully integrated monospectral camera with a noise equivalent temperature difference of $11 \mathrm{mK}$ in 2004 [5], the development of bispectral imaging systems with timely coincident detection of two different spectral bands in the MWIR was started and is now in the stage of batch production [6].

The characterization and monitoring of important fundamental electro-optical properties of IR-detector material for batch production and the results of high-performance dual-color IRcamera systems are presented in this paper.

\section{Characterization of fundamental electro- optical properties of InAs/GaSb superlattice infrared detector material}

In the following, the requirements for a successful batch production are introduced. High-quality GaSb substrates are undoubtedly essential for high detector performance in order to enable growth of epitaxial layers with low defect density. The substrate also requires an excellent quality in terms of wafer flatness, thickness variation and low dislocation density. A high band gap AIGaAsSb buffer layer is used for electrical isolation of the IR PDs from the conductive substrate. Electrical performance is routinely monitored using test structures which offer an electrical contact from the lower contact of the diode structure to the conductive substrate. For a bias voltage up to $1 \mathrm{~V}$, the measured current across the AIGaAsSb buffer layer does not exceed the resolution of the measurement setup of roughly $0.1 \mathrm{pA}$ at $77 \mathrm{~K}$, demonstrating the excellent isolating behavior of the buffer layer.

For p-i-n SL PDs, a flawless performance within the bulk of the wafer is a precondition to achieve the expected performance level of IRcamera systems. Bulk performance is monitored by various characterization techniques. Primary characterization after molecular beam epitaxy (MBE) is accomplished by the examination of the surface morphology, followed by optical wafer inspection in order to quantify surface roughness and to map the density and the location of various kinds of growth defects. This is followed by highresolution x-ray diffraction analysis, where SL period length is determined and the lattice matching to GaSb substrate and overall crystallographic quality are checked. Next, secondary-ion mass spectrometry is performed to validate doping levels and composition. For verification of the electro-optical wafer performance, quadratic mesa diodes with varying size are processed on test dies. Measurements of the dark-current density of a sufficiently large set of these diodes allow the separation of surface leakage currents from bulk-material contributions to dark current by comparing the results obtained on large-area and small-area diodes. Surface leakage currents are suppressed using a dielectric passivation. Typical values for the product of the dynamic resistance $R$ and the area $A(R A-$ product) for $p$-i-n PDs with a cutoff wavelength of $4 \mu \mathrm{m}, 5 \mu \mathrm{m}$ and $10 \mu \mathrm{m}$, found under slight reverse bias voltage at $77 \mathrm{~K}$, are $10^{8} \Omega \mathrm{cm}^{2}$, $10^{6} \Omega \mathrm{cm}^{2}$ and $10^{2} \Omega \mathrm{cm}^{2}$, respectively.

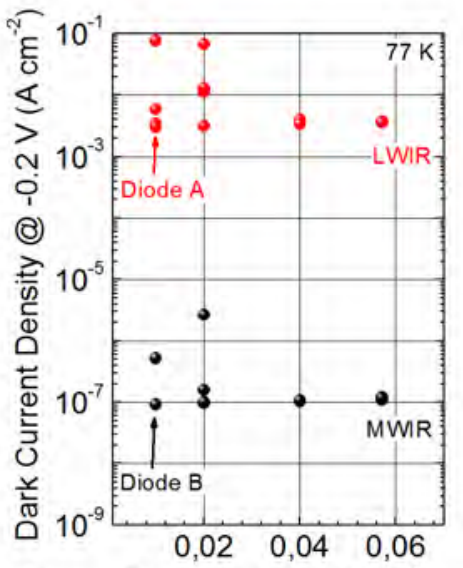

a) Perimeter / Area $\left(\mu \mathrm{m}^{-1}\right)$
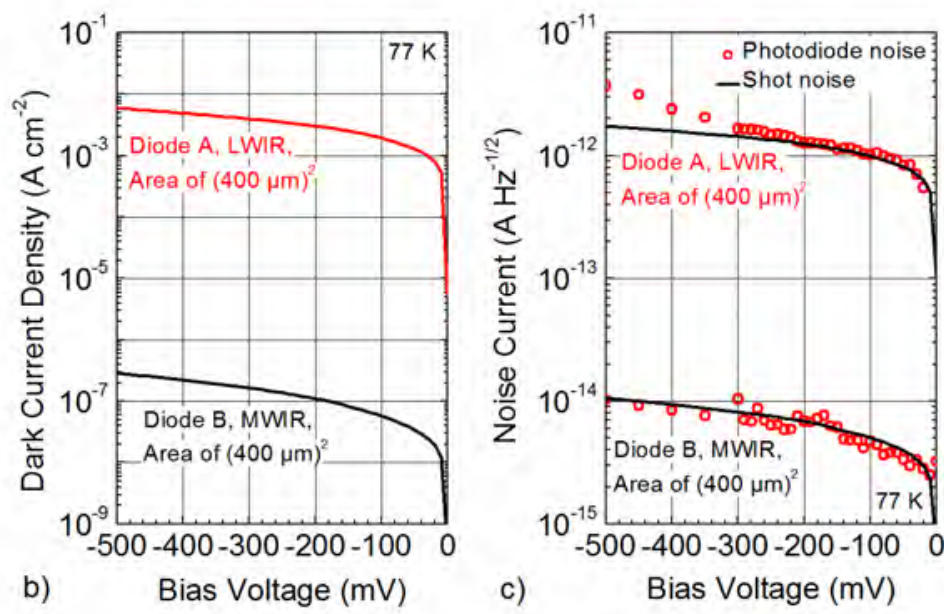

Figure 1. Dark-current density vs. perimeter-to-area ratio (a) and vs. bias voltage (b) for MWIR and LWIR photodiodes and their noise currents vs. bias voltage (c). The measured photodiode noise current accurately matches the expected shot noise according to Eq. (1). 
The dark current of a set of quadratic InAs/GaSb SL monospectral PDs with variable perimeter-to-area $(P / A)$ ratio is presented for LWIR and MWIR PDs at $77 \mathrm{~K}$. Figure 1a shows the dark-current density at a bias voltage of $0.2 \mathrm{~V}$ versus P/A-ratio. Neither in the LWIR nor in the MWIR material dark-current density depends on the P/A-ratio indicating a successful suppression of surface leakage currents. The noise currents of an LWIR and an MWIR diode, each with an area of $400 \mu \mathrm{m} \times 400 \mu \mathrm{m}$, were measured. These diodes were marked with arrows in Fig. 1a. Dark-current density versus reverse bias voltage of both diodes is shown in Fig. $1 \mathrm{~b}$. According to theory, noise associated with the dark current of a generation-recombination limited $p-i-n$ PD follows the shot noise expression

$$
I_{\mathrm{n}}^{2}=2 e\left(I_{\mathrm{d}}+2 I_{0}\right) \Delta f,
$$

where $e$ is the elementary charge, $l_{\mathrm{d}}$ the dark current, $I_{0}$ the saturation component of the diffusion current and $\Delta f$ the bandwidth of measurement [7]. Figure 1c shows noise measurements of InAs/GaSb SL homojunction p-i-n PDs which are limited by Shockley-ReadHall (SRH) processes in the space-charge region of the bulk of the material. In the whitenoise part of the spectrum, the extracted diode noise of both diodes closely matches the theoretically expected shot-noise level according to Eq. (1) [8]. Hence, noise current caused by dark current does not limit adequate InAs/GaSb SL PDs; they are rather dominated by shot noise caused by the background photon flux. Thus, they are operated under background-limited performance (BLIP) condition. The detectivity $D^{*}$, which represents the signal-to-noise ratio, is therefore given by

$$
D_{\mathrm{BLIP}}^{*}=\frac{\lambda}{h c} \sqrt{\frac{\eta}{2 \Phi_{\mathrm{B}}}},
$$

where $\lambda$ is the wavelength, $h$ is Planck's constant, $c$ is the speed of light, $\eta$ represents the $\mathrm{QE}$ and $\Phi_{\mathrm{B}}$ indicates the background photon flux density. Accordingly, detectivity is improved by increasing the quantum efficiency under BLIP operation.

The optical response is measured with mesa diodes containing an optical window in the top contact metallization. Routinely, the photoresponse spectrum delivering the cutoff wavelength and signal level is recorded. QE increases proportionally with the absorber thickness, but saturates once the absorber thickness and the electron diffusion length become comparable.

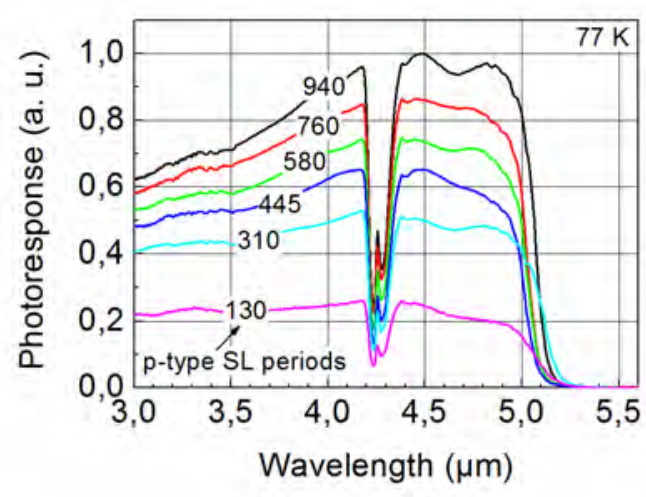

Figure 2. Photoresponse vs. wavelength for a set of six MWIR InAs/GaSb superlattice photodiodes with increasing number of p-type absorber periods.

Provided that electron diffusion length is sufficiently large, an increase of absorber layer thickness allows a theoretical QE close to unity. Figure 2 shows the responsivity which is directly proportional to the QE measured with a Fourier transform spectrometer at $77 \mathrm{~K}$ in arbitrary units in dependence of the wavelength. The signature around $4.3 \mu \mathrm{m}$ is due to carbon dioxide absorption. Up to an absorber thickness of 940 periods, no saturation of responsivity is observed. Experimentally observed photoresponse shows theoretically expected dependence on absorber thickness, when a diffusion length much beyond 940 periods is assumed, corresponding to roughly $6 \mu \mathrm{m}$ [9]. In fact, the significant rise of the photoresponse between 760 and 940 periods is a clear evidence of a diffusion length much larger than the absorber layer thickness of the thickest sample.

A very important parameter is the polarity and magnitude of the InAs/GaSb SL carrier background. Due to the very low vertical mobility of holes in InAs/GaSb SLs, a high optical PD response, as described above, is only achievable with a major contribution from highly mobile minority electrons. In a $\mathrm{p}^{+}-\mathrm{i}-\mathrm{n}^{+} \mathrm{PD}$, the thick intrinsic region should consequently be residually p-type. Carriers photogenerated in the depletion region and minority electrons excited in the neutral part of the p-type absorber contribute to the signal offering high responsivity in such a diode. The photoresponse is expected to show a nearly constant level with increased reverse bias, with only holes accelerated by the high electric field within the depletion region, giving rise to a small additional bias-dependent contribution. In contrary, a significant additional contribution which is proportional to the width of the depletion region is expected in $p^{+}-n^{-}-n^{+}$PDs. The photoresponse increases with depletion width according to $\left(V_{b i}-V\right)^{1 / 2}$, where $V_{\mathrm{bi}}$ is the 
built-in voltage and $V$ the applied bias. To determine the polarity of the carrier background in the not intentionally doped part of the $p-i-n$ $\mathrm{PD}$, we have to measure the bias dependence of the total current, which is the sum of dark current and photocurrent under high photon flux illumination. An evaluation of the signal level and the bias dependence of the signal will then give a good hint to the polarity of the background carriers. The magnitude of the carrier background is determined by $p-n$ junction capacitance-voltage analysis using the text book relation

$$
\left(\frac{A}{C}\right)^{2}=\frac{2}{\varepsilon \varepsilon_{0} q N_{\text {red }}}\left(V_{b i}-V\right) .
$$

In the case of high doping contrast at the junction, reduced carrier density $N_{\text {red }}=N_{\mathrm{A}} N_{\mathrm{D}} /\left(N_{\mathrm{A}}+N_{\mathrm{D}}\right)$ is practically equal to the concentration on the lower doped side, which is the i-layer. Fitting Eq. 3 to the lineardata regime around zero bias voltage reveals electrically active carrier background density.

A significant part of IR-camera production is the development of a contact-via technology which guarantees good electrical contact between read-out integrated circuit (ROIC) and $p$-i-n diodes. PDs require low resistance contacts to discharge the external ROIC capacitor to the level expected for the incident photon flux. The contacts to the $p-i-n$ diode are basically comprised of a highly doped semiconductor layer and a metallization sequence deposited on it. For n-type semiconductor contact layer highly Si-doped InAs is used. The p-type contact layer consists of highly Be-doped GaSb. A low-resistance ohmic contact is readily achieved with a standard $\mathrm{Ti} / \mathrm{Pt} / \mathrm{Au}$ metallization scheme. The quality of the contacts is monitored by measuring the resistance between pads with varying distance (transmission line model). Monitoring of low contact resistance is particularly important,

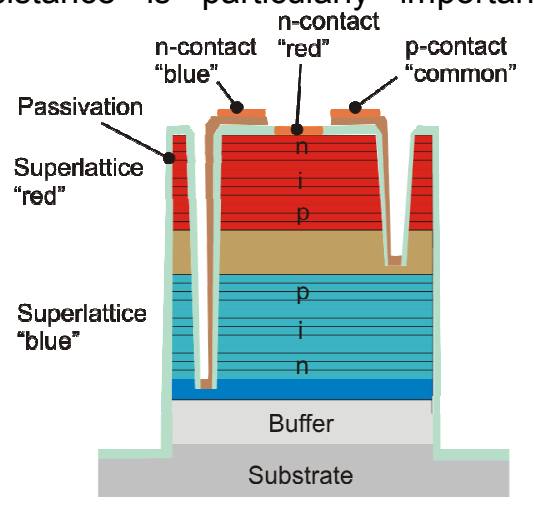

a) while using via holes in bispectral photodetectors which are described in the following.

\section{Bispectral InAs/GaSb superlattice focal plane array camera performance}

Usually, IR signatures of gases are selectively visualized utilizing narrow-band filters together with broad-band IR detection systems [10]. However, high-intense broad-band IR sources, like e.g. hot objects or solar reflections, can be misinterpreted as IR emissions from hot gases, since parts of the broad-band photon flux pass the narrow-band filter. In contrast, multispectral IR cameras differentiate between several bands in the IR range and can thus discern between various objects and gases due to their spectral signatures. A bispectral IR camera can serve as an imaging spectrometer if one channel detects the absorption / emission band of a certain molecule and the other one does not. Therefore, the manufacturing technology for the fabrication of bispectral InAs/GaSb SL detector arrays for the MWIR has been developed. MBE growth process and a reproducible process technology for front-side and back-side processing of the FPAs have been set up to facilitate the development of high-performance infrared sensors. FPAs are hybridized with custom-designed ROICs and completely integrated into full-performance IR imagers. The concept chosen for the dual-color InAs/GaSb $\mathrm{SL}$ detectors avoids any kind of temporal or spatial mismatch in the collected data, since a simultaneous, co-located registration of the two channels is realized. Figure $3 a$ shows a schematic view of a dual-color detector pixel. In each pixel, two back-to-back p-i-n InAs/GaSb SL PDs are sandwiched in between three contact layers to realize the co-located detection. The upper diode, subsequently referred to as the "red-channel" diode, senses IR radiation at $4-5 \mu \mathrm{m}$. The lower, "bluechannel" diode is sensitive at 3-4 $\mu \mathrm{m}$.

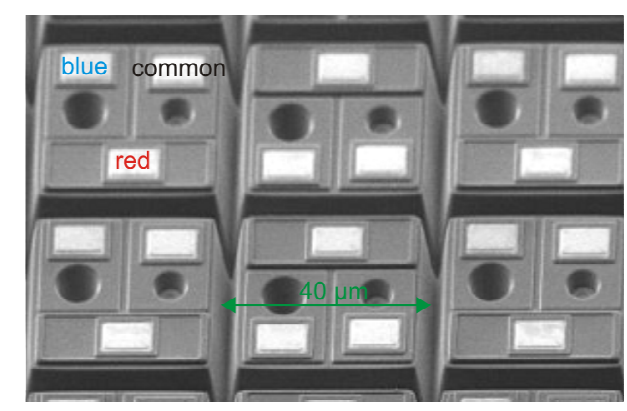

b)

Figure 3. Schematic cross section of a bispectral detector pixel (a) and SEM image of a section of a completely processed dual-color InAs/GaSb SL detector array with a pixel pitch of $40 \mu \mathrm{m}$ (b). 

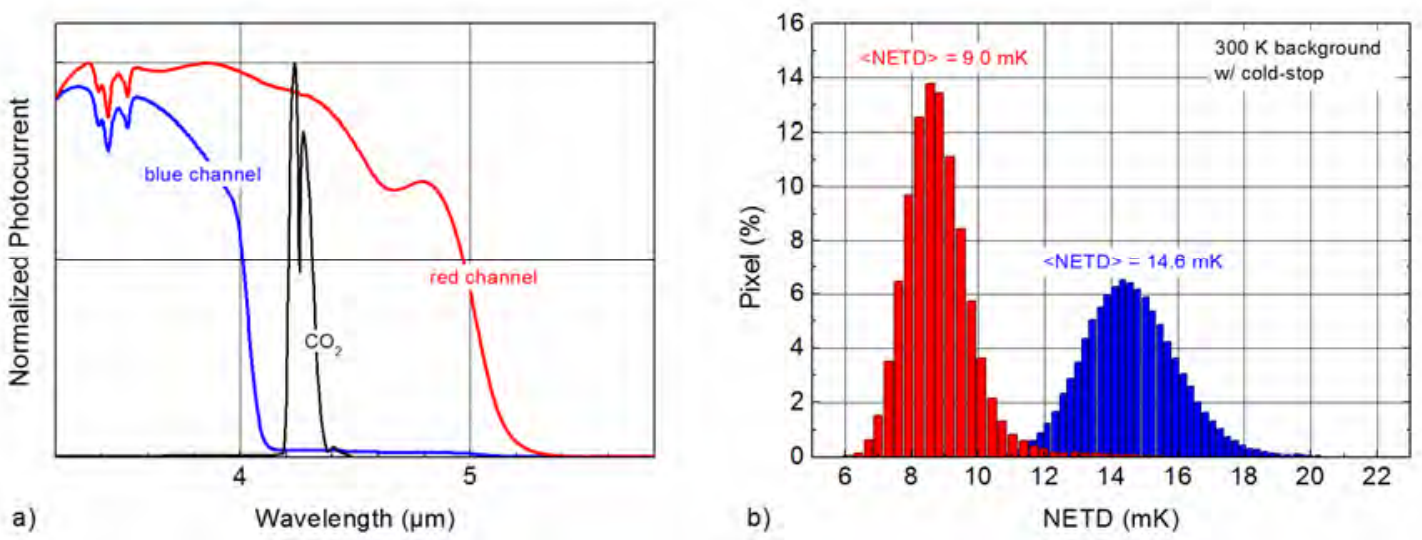

Figure 4. Normalized photocurrent spectra and the absorption spectrum of carbon dioxide [10] (a) and histograms showing the distribution of the noise equivalent temperature difference of the blue and red channel of a $288 \times 384$ dual-color InAs/GaSb superlattice detector array (b).

The p-type contact layer in between both diodes is used as the common ground and is short-circuited externally via the ROIC. The $\mathrm{p}$-in PDs supply their photoelectrons to the upper and lower n-type contact. Each diode may be individually biased by the ROIC. Beneath the lower n-type contact, a high-gap buffer layer electrically isolates the blue-channel diode from the conductive n-type substrate. For simultaneous detection of both channels, all three contact layers have to be individually connected. While the upper n-contact is directly accessible at the mesa top, each pixel requires two contact vias to the common ground and lower n-type contact. Since the pixel array is illuminated from the substrate side, free-carrier absorption in the substrate should be kept as low as possible. The concept above allows the inclusion of additional layers between the buffer and the substrate, e.g. etch stop and spacer layers, in order to facilitate the removal of the substrate either entirely or to a remaining thickness of only a few microns. In this way, the thermal stress between the Si-based ROIC and the $\operatorname{InAs} / \mathrm{GaSb} S \mathrm{SL}$ chip can be reduced. Figure $3 b$ shows a SEM image of a section of a dual-color detector array. The pixel pitch size of $40 \mu \mathrm{m}$, as well as the three contact pads on each pixel, are indicated.

In Figure $4 a$, the normalized photocurrent spectra of both, the red and the blue channel, are plotted versus wavelength. Furthermore, the spectral signature of the (0001) asymmetric stretching mode of $\mathrm{CO}_{2}[10]$ is indicated as well. Since the detection range of the red channel spans the (0001) $\mathrm{CO}_{2}$ mode in contrast to the blue channel, the red channel acts as a $\mathrm{CO}_{2}-$ sensitive channel and the blue channel as its reference channel. Thermal resolution is an important figure-of-merit for the performance of IR cameras. It is usually specified by the noise equivalent temperature resolution (NETD) representing the minimal temperature difference of a black body that can be distinguished by the detector, i.e. it yields a signal-to-noise ratio equal to one. Figure $5 b$ shows the NETD histograms for a $288 \times 384$ InAs/GaSb SL camera, operated at a temperature close to liquid-nitrogen and standard operating conditions. Mean NETD values of $14.6 \mathrm{mK}$ for the blue and $9.0 \mathrm{mK}$ for the red channel have been determined. In Figure 5 a bispectral IR image of an industrial area is shown, where the blue and red channels have been superimposed in blue and red color scales, respectively. IR radiation emitted by hot $\mathrm{CO}_{2}$ at the top of smokestacks appears in red. In contrast, broadband thermal radiation from warmer surfaces cause a brighter shade of grey, while solar scattering in clouds in the sky or solar reflections from leaves or metallic surfaces even result in a pronounced blue signal. This way, the $\mathrm{CO}_{2}$ emissions can be clearly distinguished from other IR sources which often cause false signatures in filterbased IR systems. The qualitative detection of $\mathrm{CO}_{2}$ is so sensitive that even the $\mathrm{CO}_{2}$ in the human breath can be detected.

\section{Conclusion}

We presented a bispectral infrared camera comprising two detector channels manufactured from InAs/GaSb type-II superlattices. The detection bands of the two channels range from 3-4 $\mu \mathrm{m}$ and $4-5 \mu \mathrm{m}$. This camera is thus very sensitive to carbon dioxide, enabling remote imaging on a millisecond time scale with very high thermal resolution of NETD values of $14.6 \mathrm{mK}$ and $9.0 \mathrm{mK}$ for the blue and red channel, respectively. An extensive test methodology, which includes the characterization of fundamental electro-optical properties, verifies the required functionality of InAs/GaSb superlattice infrared detector 
material being essential for the achievement of high detector performance.

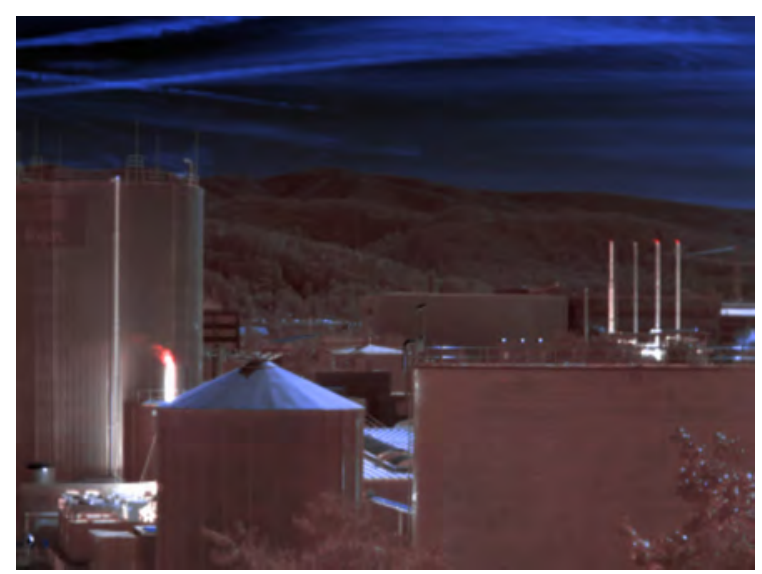

Figure 5. Bispectral IR image of an industrial area by a superposition of the two individual channels in a blue and a red color scale. The signatures of $\mathrm{CO}_{2}$ emissions of smokestacks appear in red.

\section{Acknowledgements}

The authors are grateful to F. Bernhardt, B. Campillo-Lundbeck, V. Daumer, Z. Dirani, S. Fibelkorn, M. Finck, H. Güllich, T. Henkel, S. Hugger, R. Lappe, U. Meinold. S. Rombach, and M. Wauro for assisting in MBE growth, detector processing, hybridization and characterization. Project funding by the Federal Ministry of Defense is gratefully acknowledged.

\section{References}

[1] A. Rogalski, J. Antoszewski, L. Faraone, Thirdgeneration infrared photodetector arrays, J. Appl. Phys. 105, 091101 (2009).

[2] D. L. Smith, C. Mailhiot, Proposal for strained type II superlattice infrared detectors, J. Appl. Phys. 62, 2545 (1987).

[3] F. Fuchs, U. Weimer, W. Pletschen, J, Schmitz, E. Ahlswede, M. Walther, J. Wagner, P. Koidl, High performance InAs/Ga ${ }_{1-\mathrm{x}} \ln \mathrm{x}$ Sb superlattice infrared photodiodes, Appl. Phys. Lett. 71, 3251 (1997).

[4] Y. Wei, A. Gin, M. Razeghi, G. J. Brown, Type II InAs/GaSb superlattice photovoltaic detectors with cutoff wavelength approaching $32 \mu \mathrm{m}$, Appl. Phys. Lett. 81, 3675 (2002).

[5] R. Rehm, M. Walther, J. Schmitz, J Fleissner, F. Fuchs, W. Cabanski, J. Ziegler, InAs/(Galn)Sb short-period superlattices for focal plane arrays, SPIE 5783, 123 (2005).

[6] R. Rehm, M. Walther, J. Schmitz, A. Wörl, R. Scheibner, J. Ziegler, Type-II superlattices the Fraunhofer perspective, SPIE 7660, 7660G1G12 (2010).

[7] A. van der Ziel, Proc. IEEE 58, 1178 (1970).
[8] A. Wörl, P. Kleinow, R. Rehm, J. Schmitz, M. Walther, Noise characteristics of $\ln A s / G a S b$ superlattice infrared photodiodes, Phys. Status Solidi C, in press.

[9] R. Rehm, M. Walther, J. Schmitz, F. Rutz, J. Fleissner, R. Scheibner, J. Ziegler, Status of Mid-Infrared Superlattice Technology in Germany, SPIE 7222, 72220T (2009).

[10] M. Vollmer, K.-P. Möllmann, Infrared Thermal Imaging, Ch. 7, WILEY-VCH, Weinheim, 2010. ISBN 978-3-527-40717-0. 